Cad. Est. Ling., Campinas, 53(1), Jan./Jun. 2011

\title{
TRINTA ANOS DA TEORIA CONCEPTUAL DA METÁFORA: UMA RETROSPECTIVA CRÍTICA
}

\author{
ULRIKE AGATHE SCHRÖDER* \\ UFMG
}

\begin{abstract}
RESUMO: O artigo aborda as críticas mais importantes articuladas durante os últimos trinta anos da Teoria Conceptual da Metáfora fundada por Lakoff \& Johnson. Nosssa intenção é sintetizar e discutir os pontos mais fracos da abordagem e dirigir nossa atenção para pesquisas atuais que buscam superar as falhas da teoria sem abandonar seus pressupostos básicos.
\end{abstract}

Palavras-chave metáfora conceptual, contexto, comunicação, cultura

\begin{abstract}
The paper is about the most important criticisms articulated during the last thirty years of Conceptual Metaphor Theory as established by Lakoff \& Johnson. The primary purpose is to synthesize and discuss the main weak points in order to confine our attention to current research which seeks to overcome the shortcomings of the theory without abandoning its basic assumptions.

Key-words conceptual metaphor, context, communication, culture
\end{abstract}

\section{A MUDANÇA PARADIGMÁTICA NAS PESQUISAS SOBRE A METÁFORA}

A publicação Metaphors We Live By (Lakoff \& Johnson 1980) não apenas marca o começo de uma nova orientação nas pesquisas relacionadas à metáfora, mas também fornece um alicerce fundamental para a formulação da Linguística Cognitiva que desde então assumiu influência crescente nos estudos linguísticos transdisciplinares.

Como o título programático já indica Lakoff \& Johnson distanciam-se da visão impressionista da metáfora uma vez que estas não são mais tratadas como ornamentos poéticos e retóricos da língua. Muito pelo contrário, agora, metáforas são focalizadas como expressão de estruturas conceptuais subjacentes às habilidades cognitivas do ser humano em geral de modo que a expressão metafórica representa apenas uma manifestação superficial de uma metáfora conceptual mais profunda.

Destarte, em expressões como batalha das eleições, guerra de partidos ou embate da oposição, a POLítICA é fixada pela imagem da GUERRA. Sendo assim, a metáfora permite o entendimento de um domínio abstrato ('domínio alvo') em termos de um outro, mais concreto ('domínio fonte'). Assim que as correspondências entre os dois domínios forem ativadas, automaticamente, também são mapeados os padrões de inferência inerentes aos esquemas imagéticos, ${ }^{1}$ o que é denominado 'Princípio da Invariância' por Lakoff

${ }^{*}$ UFMG/FL, Belo Horizonte (MG), Brasil. schroederulrike@gmx.com

${ }^{1} \mathrm{Na}$ TCM, 'esquemas imagéticos' são concebidos como representações esquemáticas de estruturas envolvidas em interações recorrentes com nosso meio, conferindo coerência a nossas experiências singulares, percepções sensoriais e decorrências motóricas. Um exemplo seria o esquema imagético CONTAINER que se baseia 
(1993). Portanto, a metáfora assume a função principal de organizar e categorizar nossas experiências no sentido de um universal antropológico.

Embora o livro prescinda de um encaixe histórico e teórico, de uma coerência nas referências bibliográficas e de um olhar além da produção científica anglo-americana, é recebido como "uma das novidades mais notáveis no campo da filosofia da linguagem dos últimos anos" (Burkhardt 1987, p. 42), ${ }^{2}$ uma "pedra miliária” (Lawler 1983, p. 205) e um "ensaio revolucionário sobre a metáfora" (Nuessel 1990, p. 251). Até hoje, predomina essa percepção de que a obra marca uma mudança radical.

Ora, o que, a nosso ver, finalmente, torna essa obra uma obra-chave não se revela através de sua suposta originalidade, mas sim, em sua transposição perspectival explícita e sistemática da língua à cognição e a abundância dos exemplos provindos de nossos usos cotidianos da metáfora, o que o tornou ponto de partida para o desenvolvimento de inúmeros estudos empíricos e experimentais no campo da metáfora.

Apesar desse efeito impulsivo que a Teoria Conceptual da Metáfora (TCM) exerceu sobre o desenvolvimento das pesquisas na área da Linguística Cognitiva, ao mesmo tempo, sempre foi criticada também. Na presente abordagem, nosso objetivo é sistematizar, sintetizar e apresentar as vozes críticas mais decisivas que surgiram desde o nascimento da teoria até hoje para possibilitar uma reflexão sobre os últimos trinta anos da TCM buscando por uma delineação de futuros campos de análise.

Para tal, nosso foco principal dirige-se, em especial, aos déficits teóricos imanentes e a aspectos que faltam na teoria, mas que poderiam enriquecê-la e promovê-la. Para tanto, ressaltamos que não se faz objetivo deste artigo promover discussões sobre modelos alternativos que não contemplem uma visão cognitiva da metáfora.

\section{AS PRINCIPAIS CRÍTICAS}

\subsection{Críticas relacionadas a déficits imanentes à teoria}

Um primeiro ponto de crítica refere-se à suposta originalidade da TCM que proclama o abandono das visões tradicionais, o que, de modo algum, representa uma inovação repentina como sugerida pelos próprios autores em vários momentos de suas abordagens (Lakoff \& Wehling 2008/2009, p. 14-15; Lakoff \& Johnson 1980/2003, p. xi; Lakoff 1993, p. 202; Lakoff \& Turner 1989, p. 110-139). Uma série de autores aponta a falta de contextualização historiográfica da TCM indicando as continuidades teóricas (Schmitz 1985, p. 356; Hülzer-Vogt 1987; Burkhardt 1987; Jäkel 2003; Nerlich \& Clarke 2001; Schröder 2004; 2008; 2009b). Linz (2004, p. 254) discorre sobre um "gesto da renovação revolucionária exigida pela Linguística Cognitiva", ${ }^{5}$ que já se torna duvidosa

\footnotetext{
em nossa experiência de olhar para dentro de caixas ou quartos, encher copos de água, tirar objetos de um armário e de uma bolsa, entrar e sair de casas, comer e beber etc. (Johnson 1987,pp. 19-30).

2 "eine der bemerkenswertesten sprachphilosophischen Neuerscheinungen der letzten Jahre"

3 "milestone"

4 "groundbreaking essay on metaphor"

5 "Gestus der revolutionären Erneuerung, den die Kognitive Linguistik für sich in Anspruch nimmt"
} 
por considerar apenas a antiguidade. Enquanto leituras profundas comprovam que até Quintiliano e Aristóteles já estavam plenamente conscientes da figuratividade presente quase totalmente em nossa fala.

A maioria das ideias da obra e até boa parte dos exemplos pode ser encontrada em estudos antecessores, que surgem desde o século dezessete em abordagens filosóficas (Clauberg, Vico, Locke, Leibniz, Lambert, Kant, Nietzsche, Mauthner, Vaihinger, Richards, Blumenberg), antropológicas (Herder, Boas, Sapir, Whorf, Gehlen), psicológicas (Stählin, Bühler) e linguísticas (Paul, Wegener, Gerber, Lady Welby, Black, Weinrich), para apenas nomear os mais importantes precursores esquecidos (Schröder 2008a).

Muitas discussões decorrem de indeterminações, vaguezas, incoerências e inferências precipitadas, colocando em discussão postulados fundamentais imanentes à teoria. Linz (2002, p.100-101) acusa os autores da falta de consistência com relação à terminologia desenvolvida. Assim, por exemplo, os autores não esclarecem o status dos 'esquemas imagéticos': são inatos ou adquiridos ontogeneticamente, preconceptual ou conceptual? De modo similar, também a assim chamada 'built-in logic' (Lakoff 1989, 115) dos esquemas imagéticos parece problemática epistemologicamente.

A mesma repreensão vale para a noção dos 'modelos cognitivos idealizados' ${ }^{6}$ Até o próprio Lakoff (1987, p. 284) percebe esse problema quando confessa que é certo que ele gostaria de ilustrar como ele mesmo imaginaria modelos cognitivos idealizados; não obstante, não pretende esboçar definições detalhadas.

Em seu ensaio polêmico, Haser (2005) busca romper com a posição filosófica ecleticista e simplicista de Lakoff \& Johnson. Entretanto, recomenda-se análise cautelosa dessa crítica, pois em muitos momentos, a mesma não ultrapassa a discussão sobre certas 'batalhas de posições' conhecidas. A acusação da teórica (Haser 2005, p. 249) de que os autores reduziriam metáforas e metonímias a estratégias cognitivas, ao invés de descrevêlas como fenômeno da língua, é redundante, pois é exatamente isso o que os autores querem comprovar: a metáfora, para eles, vai além de uma mera expressão na superfície da língua. E uma das evidências bastante convincentes são os inúmeros exemplos que ilustram como uma série de expressões metafóricas distintas provém do mesmo campo semântico.

Outro ponto de crítica refere-se a um detalhe da teoria de Lakoff e Johnson, denominado por Jäkel (2003, p. 41) como 'Tese da Unidirecionalidade' .Jäkel assim o denomina com base na afirmação dos autores de que mapeamentos metafóricos sempre percorrem de domínios concretos a domínios abstratos. Nisso, Jäkel (2003, p. 5859) observa um 'fisiocentrismo' e um 'antropocentrismo' que pretende testar. Em um experimento realizado pelo autor (Jäkel 2003, p. 63-84), 39 sujeitos classificaram dez metáforas inventadas, de acordo com o grau de dificuldade apresentado para entedêlas. De fato, grosso modo, o experimento corroborou a tese de Lakoff \& Johnson, pois

${ }^{6}$ O termo 'modelos cognitivos idealizados' (Lakoff 1987, p. 68-76) refere-se a modelos abstraídos da realidade sócio-cultural como representações de experiências recorrentes com validade intersubjetiva, exigindo um status semelhante a teorias. Formam um tipo de conhecimento de fundo para nossa atuação no mundo. Por exemplo, um conceito como 'terça-feira' apenas faz sentido perante o fundo do modelo cognitivo idealizado 'semana', e 'semana é idealizada contando que não exista objetivamente, mas representa um construto culturalmente ancorado. 
as metáforas com domínios fontes mais abstratos foram também consideradas como as mais difíceis no que tange à compreensão. Porém, Jäkel alerta sobre uma generalização absoluta desse princípio, uma vez que há muitos exemplos invertidos que, não obstante, são compreensíveis para todos como, por exemplo, a afirmação famosa de Friedrich Engels: "Na família, o homem é o burguês e a mulher o proletário."7 Outros exemplos são apresentados por Alverson (1991) e provêm de domínios abstratos como ECONOMIA, o que se reflete, entre outros, na expressão "Eu conto com ele". Alverson também põe em dúvida a recondução de todas as metáforas a esquemas imagéticos, uma réplica que também ganha espaço novamente em discussões atuais (Zinken \& Musolff 2009). Assim, o teórico é capaz de ilustrar que há, por exemplo, muitas expressões metafóricas descendendo do domínio PÔQUER como em "Vamos colocar nossas cartas na mesa" (Alverson 1991, p. 101-102). Tais expressões não podem mais ser decompostas para primitivos semânticos associáveis a esquemas imagéticos, de modo que elas melhor correspondem às 'categorias básicas', segundo Rosch (1978), do que a gestalts abstratos.

Pode-se acrescentar outro aspecto não considerado na 'Tese da Unidirecionalidade', a saber, o processo metafórico; uma vez que a unidirecionalidade proclamada parte de uma perspectiva evolucionista excluindo a pergunta sobre o que acontece assim que dois domínios fossem interligados no uso da língua atual. Respostas detalhadas são dadas por uma corrente paralela aos estudos da metáfora, pela escola interacionista (Stählin 1913; Bühler 1934/1984; Richards 1936/1965; Black 1962) e pelo seu sucessor, a Teoria da Mesclagem (Fauconnier \& Turner 2002).

Logo após a publicação de Metaphors We live By, Holland (1982) e Butters (1981) questionam se domínios fonte como GUERRA realmente estariam mais próximos à experiência do que o domínio alvo, por exemplo, DISCUSSÃo; uma vez que a maioria das pessoas conheceriam 'discussões' através da própria experiência cotidiana, ao passo que 'guerra', para muitos, seria algo que se compreende através de relatos de terceiros, como os meios de comunicação em massa, por exemplo. Além disso, os autores também suspeitam da suposição de que o domínio fonte sempre seja de natureza física, contrariamente, atribuem um alto grau de culturalidade a essa questão. De fato, confrontos físicos como se encontram no reino animal já não se associam mais àquilo que se denomina 'guerra' atualmente. Contudo, o próprio Lakoff (1982) esclarece que considera a proclamada proximidade à experiência de modo não ontogenético, mas sim, filogenético em termos de um estoque de conhecimento culturalmente transmitido, remetendo às 'metáforas fundadas indiretamente' como “metáforas da forma $A$ é $B$, no qual $B$ é concebido majoritariamente por meio de metáforas culturais e convencionais do que por meio de experiência direta" (Lakoff 1982, p. 5). ${ }^{8}$ Como consequência, posteriormente, Lakoff substitui a metáfora conceptual DISCUSSÃO É GUERRA pela metáfora DISCUSSÃO É CONFLITO FÍSICO (Lakoff \& Wehling 2008/2009, p. 20). Porém, Rolf (2005, p. 241) aponta o fato importante de que esta concessão sobre as experiências indiretas da guerra implica que, aparentemente, também formamos metáforas com base em experiências interpessoais que, por sua vez,

\footnotetext{
7 "In der Familie ist der Mann der Bürger und die Frau der Proletarier."

8 "metaphors of the form $A$ is $B$, where $B$ is understood via cultural conventional metaphors rather than via direct experience"
} 
sempre e, somente, podem ser experiências comunicadas verbalmente. Portanto, não é necessariamente a experiência individual e imediata que serve como base para nossas metáforas conceptuais mas sim, da mesma forma, a interação verbal e a vivência cultural podem representar o ponto de partida para nossas construções metafóricas do mundo social.

Uma série de contraposições questiona inferências distintas da teoria: Jackendoff (2002, p. 356-357) ilustra a diversidade de projeções conceptuais que se encontram ocultas em frases como "O mensageiro foi de Paris para Istambul"9 (mudança de local), "A herança, finalmente, foi para Fred"10 (mudança de proprietário) e "A luz foi de verde para vermelho"11 (mudança de característica), mas ele chega a outra conclusão, diferente daquela de Lakoff \& Johnson. Enquanto a TCM interpreta essa polissemia como derivação, Jackendoff a percebe como instância paralela de um esquema mais abstrato. No entanto, a posição defendida por Jackendoff continua especulativa uma vez que sua proposta alternativa também parte de abstrações conceptuais superordenadas, procedendo, por conseguinte, tão dedutivamente como aquela de Lakoff \& Johnson.

Para outros críticos (Hülzer-Vogt 1987, p. 246; Haser 2005, p. 179-180), não há plausibilidade justificada para a ligação criada entre a expressão metafórica e a metáfora conceptual subjacente. Steen (2007, p. 135) indaga qual seria o critério para decidir se expressões como "Tenho que orçar meu tempo"12 ou "Gastei tempo demais nisso"13 baseiam-se na metáfora conceptual TEMPO É DINHEIRO Ou TEMPO É UM RECURSO VALIOSO. Vervaeke \& Kennedy (1996) questionam a tese de que expressões metafóricas sempre poderiam ser reconduzidas a uma metáfora conceptual implícita por demonstrar que há muitas expressões que são idiomáticas e inflexíveis. Inferir de expressões idiomáticas, como em: come into money ou fall into debt ${ }^{14}$, a existência de uma metáfora conceptual como DINHEIRO É UM LUGAR contrariaria o fato de que tais expressões não podem ser variadas com outras palavras do mesmo domínio fonte. Ademais, os autores demonstram que uma palavra como attack poderia ser usada ao invés das expressões assault e criticize; todavia, assault e criticize, por sua vez, não são equivalentes. Pode-se concluir que attack assume dois significados distintos, o que coloca em dúvida se realmente tal fenômeno ainda poderia ser nomeado como 'metáfora' ou se seria mais adequado constatar que agora, a expressão tenha obtido um significado abstrato independente.

Da mesma forma, a delineação à metonímia é contestável. O capítulo sobre a metáfora THINKING IS PERCEIVING do livro Philosophy in the Flesh, é introduzido pela frase "Recebemos a maioria do nosso conhecimento através de visão"15 (Lakoff \& Johnson 1999 , p. 238). Ora, Steen $(2007$, p. 219) pergunta por que os autores não optaram por descrever este conceito como metonímico no qual o domínio da percepção destaca o aspecto do pensamento dentro do cenário da aquisição do conhecimento.

\footnotetext{
9 "The messenger went from Paris to Istanbul"

10 "The inheritance finally went to Fred"

11 "The light went from green to red"

12 "I have to budget my time"

13 "I spent too much time on that"

${ }^{14}$ Os exemplos não foram traduzidos por serem expressões idiomáticas do inglês.

15 "We get most of our knowledge through vision"
} 
Murphy (1996) inicia uma discussão que se estabelece por volta do dualismo entre 'visão forte' e 'visão fraca': a visão forte da TCM afirma que não conseguimos falar e nem refletir sobre domínios abstratos ou emocionais sem a ajuda de domínios concretos baseados na experiência. Segundo essa posição, metáforas tornam-se 'veículos do pensamento' (Murphy 1996, p. 178) como o 'Pincípio da Invariância' constata, embora por outro lado, até o próprio Lakoff assinale que os possíveis mapeamentos sejam restringidos pela estrutura do domínio alvo; com toda razão, Murphy vê uma contradição nisso. A despeito disso, a versão fraca concede uma estrutura própria ao domínio alvo.

Finalmente, muitos autores dedicam-se a falhas metodológicas, entre eles, representantes da linguística de corpus, cujas críticas se direcionam aos exemplos de Lakoff \& Johnson por estes não serem retirados de corpora autênticos (Charteris-Black 2004; Deignan 2005; Stefanowitsch \& Gries 2007; Sardinha 2007). Sardinha (2007, p. 139-140) consegue comprovar essa artificialidade dos exemplos por meio de uma pesquisa na internet revelando um resultado elucidativo: a frase apresentada por Lakoff \& Johnson "It's hard to get that idea across to him" apenas se encontra 14 vezes na internet $\mathrm{e}$ todos os 14 tokens apresentam reproduções do exemplo dos autores. „Your reasons come through to us" mostra 26 ocorrências, mas também somente se trata de cópias do exemplo original de Lakoff \& Johnson. No caso do exemplo "Your claims are indefensible", há até 220 ocorrências das quais 207 (94\%) representam referências ao exemplo usado por Lakoff \& Johnson. Esta documentação de Sardinha ilustra muito bem a impossibilidade em desconsiderar o uso em detrimento da criação de exemplos meramente introspectivos, uma vez que tal conduta não garante de modo nenhum a autenticidade das expressões. Os trabalhos pioneiros de Alice Deignan (2005) com base no Bank of English corpus levaram a um questionamento das hipóteses da TCM porque conseguiram detectar que os respectivos padrões da língua, no uso concreto, por um lado, são mais dinâmicos e, por outro lado, são mais específicos. Quando se analisa, por exemplo, a metáfora conceptual SISTEMAS ABSTRATOS COMPLEXOS SÃO PLANTAS, como descrita pela TCM, observa-se que, por meio de uma revisão detalhada com ajuda de dados de corpora, certas expressões somente são usadas para certos subdomínios: por exemplo, empresas ou negócios podem florescer mas não murchar (Deignan 2005, p. 176). Uma palavra como rocha (rock), no singular, metaforicamente, comporta-se diferentemente no plural. No singular, na maiora das vezes, sua conotação é positiva como no exemplo "the rock on which our society is built"16. Todavia, no plural, a conotação tende a ser negativa e interligada à metáfora da VIAGEM como na frase "The marriage has been on the rocks for a while" (Deignan 2005, p. 157-159). Igualmente, flame (chama), no singular, tipicamente serve para a construção de domínios alvo positivamente conotados como festas, ao passo que o plural já se aplica a contextos ameaçadores e incontroláveis. Um estudo de corpus em que Semino (2008, p.191-196) analisou 139 concordâncias distintas para a palavra rich revelou que os significados contextuais, principalmente, referem-se à abundância, à multiplicidade ou à intensidade, de modo que se tornam duvidosas as inferências da TCM de que frases como "Ele teve uma vida rica" poderiam servir como evidência para a metáfora conceptual

\footnotetext{
${ }^{16}$ Os exemplos não foram traduzidos por serem mais características para a língua inglesa.
} 
Cadernos de Estudos Lingüísticos, Campinas, 53(1), Jan./Jun. 2011

profunda UMA VIDA DE OBJETIVOS É NEGÓCIO. Além disso, a teórica também aponta que em inglês britânico, a colocação rich life é pouco usada.

\subsection{Críticas relacionadas a aspectos abandonados pela teoria}

Uma segunda linha de críticas tem uma relevância alta para o desenvolvimento de correntes atuais (Schröder 2008b, 2008c). Esta linha pergunta pelos fatores socioculturais, contextuais e funcional-comunicativos, que, para muitos pesquisadores do campo da análise do discurso (Cameron 2007, 2008) e do campo das comparações culturais (Kövecses 2005; Zinken 2004; Fernandez 1991, Kimmel 2005; Schröder 2008b; 2008c) foram excluídos pela primeira geração, em vista da prioridade atribuida às metáforas conceptuais idealizadas de Lakoff \& Johnson. Observando-se a cadeia monodirecional partindo da experiência sensomotora, passando pelos esquemas imagéticos e pelos conceitos abstratos, chegando às expressões da língua, continua sendo opaco como, por fim, as descontinuidades entre os indivíduos e as comunidades de fala poderiam ser explicadas (Alverson 1991, p. 111; Zinken 2004, p. 131). Alguns autores ressaltam que os exemplos de Lakoff \& Johnson provêm exclusivamente da língua inglesa. É com base nesses exemplos que os autores proclamam a universalidade de certos conceitos metafóricos. Em sua introdução à coletânea de estudos antropológicos sobre a metáfora, Fernandez (1991, p. 2) já nota que os estudos sobre a metáfora, no campo da antropologia, priorizam outros aspectos "em que o trabalho pragmático e coincidente de figuras do pensamento dentro de estruturas sociais e visões do mundo culturais é o referente primordial para o entendimento teórico". ${ }^{17}$ A exigência da universalidade da metáfora histórica não pode ser realizada consistentemente:

\footnotetext{
"Metáforas conceptuais dos domínios movimento, ação, vida, interação etc. paralisam os universais históricos, embora seja justamente interessante observar até que ponto elas mesmo são parte de discursos crescidos historicamente e esquemas imagéticos culturalmente transmitidos."18 (Debatin 1995, p. 247)
}

Reparos semelhantes encontram-se na reflexão de Feilke (1994, p. 41) que critica que "a fronteira, entre, por um lado, as condições cognitivas-conceptuais da motivação e, por outro lado, a formação convencional da competência e do conhecimento semântico através do falar, não é considerada suficientemente. $\mathrm{O}$ fato de que há uma formação estrutural emergente por meio de comunicação não chega no foco da atenção." 19

E não é apenas o contexto sociocultural que rompe com as explicações unidirecionais. É a posteriorização da língua em si que também parece duvidosa. Por conseguinte,

17 "the pragmatic and coincidental work of figures of thought within social structures and cultural worldviews is the primary referent of theoretical understanding"

18 "Konzeptuelle Metaphern aus den Bereichen Bewegung, Handlung, Leben, Interaktion etc. erstarren zu ahistorischen Universalien, obwohl doch gerade bei ihnen zu untersuchen wäre, inwieweit sie selbst Teil historisch gewachsener Diskurse und kulturell tradierter Bildfelder sind."

19 "die Grenze zwischen den kognitiv-konzeptuellen Motivierungsverhältnissen einerseits und der konventionellen Formierung der Kompetenz und des semantischen Wissens durch das Sprechen andererseits nicht ausreichend berücksichtigt wird. Die Tatsache einer emergenten Strukturbildung durch Kommunikation kommt nicht zu ihrem Recht." 
cognição e língua não podem ser entendidas como sequência, mas sim, como interação de modo bidirecional (Leezenberg 2001, p. 145). Melo Mouro (2005) parte da hipótese de que, para interpretar uma metáfora, os falantes não apenas procedem do mapeamento entre domínios conceptuais distintos como proposto por Lakoff \& Johnson, mas também levam em conta a estrutura semântica dos itens lexicais que ocupam as funções de tópico e veículo de uma metáfora. Ele chega à seguinte conclusão: “Assim, a metáfora conceptual não é logicamente anterior à metáfora lingüística, mas está conectada com ela" (Melo Mouro 2005, p. 110).

Além disso, com essa suposição da monodirecionalidade, a TCM desagua em uma série de aporias como se reflete, entre outros, na contradição que há entre os termos 'metáfora conceptual' e 'metáfora convencional'. A noção da convencionalidade implica na dependência cultural das metáforas conceptuais, mas como a convencionalidade pode ser produzida se o processo linguístico é visto como secundário? Segundo Leezenberg (2001, p. 144), seria impossível imaginar categorias básicas como 'mãe' ou 'mesa' como conceitos que antecedem sua expressão na língua. Aparentemente, há um paradoxo aqui: embora o conceito experientalism com sua fundamentação na ação e cultura representa a palavra-chave contra o paradigma gerativista, Lakoff \& Johnson marginalizam a performance linguística de modo que, eliminando as funções comunicativas da língua, chega-se a um conceito aporético de uma convencionalidade não-discursiva (Linz 2004, p. 256-257). Nisso, signos linguísticos são reduzidos a expressões que apenas etiquetam conceitos extralinguísticos. Como Lakoff \& Turner $(1989,109)$ frisam: "Palavras são sequências de som que, convencionalmente, exprimem conceitos." ${ }^{20}$

Além desses problemas epistemológicos causados pelos conceitos pré-linguísticos pertencentes a sujeitos autônomos, a falta do interesse na língua descuida os fatores funcionais da metáfora no seu contexto do uso. Como Linz (2002, p. 255) afirma, a TCM não consegue superar a perspectiva solipsista do conhecimento linguístico individual em termos de um sistema cognitivo internalizado, o que deixa intacta a dicotomia chomskiana da competência e performance abandonando a função comunicativa. Com isso, observase novamente uma descrição estática da língua partindo de um falante idealizado, desconsiderando a língua como processo:

\begin{abstract}
“Observando a abordagem de Lakoff, mostra-se [...] que não apenas a função cognitiva da língua é marginalizada, também sua função comunicativa é eliminada. Isso supreende mais ainda tomando em consideração que os representantes da 'Linguística Cognitiva', em oposição ao paradigma cognitivista, realçam a dependência da experiência e a condicionalidade cultural da cognição. É certo que a construção do significado é rotulado como processo interativo; não obstante, não é analisada sob o ângulo de um processo comunicativo. Os contextos experimentais, considerados tão centrais para a categorização e a construção de sentido, no caso de Lakoff e Johnson, delimitamse a interações monólogas dos sujeitos com seu meio sem que aspectos dialógicos ou comunicativos sejam considerados."21 (Linz 2002, p. 152)
\end{abstract}

20 "Words are sound sequences that conventionally express concepts."

21 "Betrachtet man den Forschungsansatz von Lakoff, so zeigt sich [...], daß nicht nur die kognitive Funktion von Sprache marginalisiert wird, sondern auch ihre kommunikative Funktion einer weitgehenden Eliminierung anheimfällt. Dies erstaunt umso mehr, als die Vertreter der, Cognitive Linguistics in Abgrenzung zum kognitivistischen Paradigma gerade die Erfahrungsabhängigkeit und kulturelle Bedingtheit der Kognition hervorheben. Die Konstruktion von Bedeutung wird zwar als interaktiver Prozeß ausgewiesen, aber dennoch nicht 
De modo similar, Gibbs exige uma nova direção para os estudos no campo da TCM o que se reflete no título programático de um artigo publicado em 1999: Taking metaphor out of our heads and putting it into the cultural world (Gibbs 1999).

No que diz respeito à exclusão da dependência do ponto de vista relativista, Sinha \& Jensen de López (2000) comparam Lakoff \& Johnson com Piaget que, ao contrário do seu colega russo Vygotsky, continua preso a um individualismo epistémico por descuidar do contexto sociocultural e comunicativo do desenvolvimento cognitivo.

Destarte, a tese da corporificação, a saber, a ideia de que a orientação sensomotórica do ser humano determine a estrutura do pensar e, por conseguinte, do falar, sofre dos mesmos déficits porque somente rompe com metade do paradigma cartesiano: ela consegue vencer o dualismo entre corpo e mente; todavia, por outro lado, deixa intacto o antagonismo entre indivíduo e sociedade. Assim, os autores percebem o perigo de um certo 'solipsimo neural' (Sinha \& Jensen de López 2000, p. 20).

Pode-se acrescentar mais reflexões: de uma perspectiva comunicativa, há que se questionar se, em situação autêntica de comunicação, seria realmente possível distinguir entre domínios nitidamente separados, ou se a abordagem dedutiva de Lakoff \& Johnson, de fato, apresenta uma perspectiva macro descontextualizada que não consegue considerar a multidimensionalidade do processo comunicativo online. Sendo assim, fora as funções epistemológica e cognitiva, as funções expressiva, apelativa, metacomunicativa, estética, fática, ilustrativa, argumentativa e sócioregulativa são excluidas da análise.

Ademais, perante a concentração na metáfora 'instalada' no nível cognitivo, os problemas de compreensão, que fazem parte da comunicação cotidiana, são apagados.

\section{CONCLUSÃO E PANORAMAS PARA O FUTURO}

Nossa retrospectiva das críticas principais que se estabeleceram junto à publicação do clássico Metaphors We Live By até hoje, trinta anos depois, não teve como intuito uma anulação da teoria. Muito pelo contrário, pretendeu-se ilustrar que uma avaliação feita de um certo ponto de vista observador sempre teria que tomar em consideração os dois lados.

Uma vez que o sucesso constante da teoria também se reflete até no boom atual e constante da TCM e da Linguística Cognitiva, considerou-se necessário aproveitar seus trinta anos para refletir sobre as limitações e algumas visões restritas inerentes à mesma..

Um olhar retroativo, ao mesmo tempo, aponta as perspectivas para o futuro. De fato, já se observa um certo advento para novos rumos. Percebe-se, por um lado, um interesse crescente pela dinamização da metáfora e por seu processamento online nos estudos sobre a mesclagem metafórica (Fauconnier \& Turner 2002) e a extensão dessa teoria por uma revisão, integrando fatores contextuais e semióticos (Brandt \& Brandt 2005).

Por outro lado, enquanto esta linha ainda continua muito presa ao indivíduo que constrói seu mundo cognitivo solipsisticamente, formam-se cada vez mais estudos

unter der Perspektive eines kommunikativen Vorgangs betrachtet. Die für Kategorisierung und Sinnerzeugung als so zentral erachteten Erfahrungskontexte beschränken sich etwa bei Lakoff und Johnson auf monologische Interaktionen der Subjekte mit ihrer Umwelt, ohne daß dialogische oder kommunikative Aspekte mit in den Blick genommen würden." 
voltados para a análise da metáfora em seu uso na língua (Cameron 2007, 2008; Semino 2008; Cameron \& Deignan 2003; Steen 2007; Hülzer-Vogt 1991; Holt \& Drew 2005; Ponterotto 2003; Schröder 2007; Zanotto \& Palma 2008) modificando a terminologia da primeira geração rumo a uma perspectiva mais comunicativa e funcional.

Finalmente, observa-se um aumento significativo no número de pesquisas no campo comparativo relativizando os proclamados universais e encaixando as metáforas em seu contexto cultural (Kövecses 2005; Schröder 2009a, 2009b; Silva Sinha et al. 2009; Kimmel 2005; Quinn 1991).

\section{REFERÊNCIAS BIBLIOGRÁFICAS}

ALVERSON, H. (1991). "Metaphor and Experience: Looking Over the Notion of Image Schema". In: FERNANDEZ, J. W. Beyond metaphor: The theory of tropes in anthropology. Stanford: Stanford University Press, pp. 94-117.

BLACK, M. (1962). Models and Metaphors. Ithaca, New York: Cornell University Press.

BRANDT, L. \& BRANDT, Per Aage. (2005). "Making sense of a blend. A cognitive-semiotic approach to metaphor". Annual Review of Cognitive Linguistics 3, pp. 216-249.

BÜHLER, K. (1934/1982). Sprachtheorie: die Darstellungsfunktion der Sprache. Stuttgart: Fischer.

BURKHARDT, A. (1987). "Wie die , wahre Welt endlich zur Metapher wurde. Zur Konstitution, Leistung und Typologie der Metaphe”. In: Conceptus XXI, 52, pp. 39-67.

BUTTERS, R. R. (1981). Do ,Conceptual Metaphors Really Exist?“ In: Southeastern Conference on Linguistics Bulletin 5, 3, pp. 108-117.

CAMERON, L. "Metaphor and talk". (2008) In: GIBBS, R. W. Jr. The Cambridge Handbook of Metaphor and Thought. Cambridge: Cambridge University Press, pp. 197-211.

. (2007). "Confrontation or complementarity? Metaphor in language use and cognitive metaphor theory". In: Annual Review of Cognitive Linguistics 5, pp. 107-135.

CAMERON, L. \& DEIGNAN, A. (2003). "Combining Large and Small Corpora to Investigate Tuning Devices Around Metaphor in Spoken Discourse”. In: Metaphor And Symbol 18 (3), pp. 149-160.

CHARTERIS-BLACK, J. (2004). Corpus approaches to critical metaphor analysis. Basingstoke: Palgrave Macmillan.

DEBATIN, B. (1995). Die Rationalität der Metapher. Berlin, New York: Walter de Gruyter.

DEIGNAN, A. (2005). Metaphor and Corpus Linguistics. Amsterdam: John Benjamins.

FAUCONNIER, G. \& TURNER, M. (2002). The Way We Think. New York: Basic Books.

FEILKE, H. (1994). Common sense-Kompetenz. Überlegungen zu einer Theorie "sympathischen" und "natürlichen" Meines und Verstehens. Frankfurt: Suhrkamp.

FERNANDEZ. J. W. (1991). "Introduction: Confluents of Inquiry”. In: FERNANDEZ, J. W. Beyond metaphor: The theory of tropes in anthropology. Stanford: Stanford University Press, pp. 1-13. 
Cadernos de Estudos Lingüísticos, Campinas, 53(1), Jan./Jun. 2011

GIBBS, R. W. Jr. (1999). “Taking metaphor out of our heads and putting it into the cultural world”. In: GIBBS, R. W. Jr. \& STEEN, G. J. Metaphor in Cognitive Linguistics. Amsterdam, Philadelphia: John Benjamins Publishing Company.

HASER, V. (2005). Metaphor, Metonymy, and Experientialist Philosophy: Challenging Cognitive Semantics. Berlin: Mouton de Gruyter.

HOLLAND, D. (1982). "All is Metaphor: conventional Metaphors in Human Thought and Language". In: Reviews in Anthropology, 9, pp. 287-297.

HOLT, E. \& DREW, P. (2005). "Figurative Pivots: The Use of Figurative Expressions in Pivotal Topic Transitions". In: Research on Language and Sozial Interaction, 38, 1, pp. 35-61.

HÜLZER-VOGT, H. (1991). Kippfigur Metapher-metaphernbedingte Kommunikationskonflikte in Gesprächen. Ein Beitrag zur empirischen Kommunikationsforschung. Band 1: Gesprächsanalyse. Münster: Nodus Publikationen.

. (1987). Die Metapher. Kommunikationssemantische Überlegungen zu einer rhetorischen Kategorie. Münster: Nodus Publikationen.

JACKENDOFF, R. (2002). Foundations of language: Brain, meaning, grammar, evolution. Oxford: Oxford University Press.

JÄKEL, O. (2003). Wie Metaphern Wissen schaffen: die kognitive Metapherntheorie und ihre Anwendung in Modell-Analysen der Diskursbereiche Geistestätigkeit, Wirtschaft, Wissenschaft und Religion. Hamburg: Kovač.

JOHNSON, M. (1987). The Body in the Mind: The Bodily Basis of Meaning, Imagination, and Reason. Chicago: University of Chicago Press.

KIMMEL, M. (2005). "Culture regained: situated and compound image schemas". In: HAMPE, B. \& GRADY, J. E. From Perception to Meaning. Image Schemas in Cognitive Linguistics. Berlin, New York: Mouton de Gruyter, pp. 285-311.

KÖVECSES, Z. (2005). Metaphor in Culture. Universality and Variation. Cambridge: Cambridge University Press.

LAKOFF, G. (1993)."The contemporary theory of metaphor". In: ORTONY, A. Metaphor and Thought. Cambridge: Cambridge University Press, pp. 202-251.

. (1989). "Some empirical results about the nature of concepts". Mind and Language 4, pp. 119-154.

(1987). Women, Fire and Dangerous Things: What Categories Reveal about the Mind. Chicago: University of Chicago Press.

. (1982). Categories and Cognitive Models. Berkeley cognitive Science Report, 2.

LAKOFF, G. \& JOHNSON, M. (1999). Philosophy in the Flesh. The Embodied Mind and its Challenge to Western Thought. New York: Basic Books.

. (1980/2003). Metaphors We Live By. Chicago: The University of Chicago Press.

LAKOFF, G. \& TURNER, M. (1989). More than Cool Reason. A Field Guide to Poetic Metaphor. Chicago, London: The University of Chicago Press.

LAKOFF, G. \& WEHLING, E. (2009). Auf leisen Sohlen ins Gehirn. Politische Sprache und ihre heimliche Macht. Heidelberg: Carl-Auer-Systeme.

LAWLER, J. M. (1983 ). “Review: Metaphors We Live By”. In: Language, 59/1, pp. 201-207. 
SCHRÖEDER - Trinta anos da teoria conceptual da metáfora...

LEEZENBERG, M. (2001). Contexts of Metaphor. Amsterdam: Elsevier.

LINZ, E. (2004 ). „Sprachlose Metaphern. Zur Rhetorizität der Kognition und ihrer Modellierung in der kognitiven Linguistik“. In: FOHRMANN, J. Rhetorik. Figuration und Performanz. Stuttgart, Weimar: Metzler. pp. 246-266.

. (2002). Indiskrete Semantik. Kognitive Linguistik und neurowissenschaftliche Theoriebildung. München: Wilhelm Fink Verlag.

MELO MOURA, H. M. (2005). "Metáforas e Regularidades Lingüísticas”. In: MIRANDA, N. S. \& NAME, C. (Ed.). Lingüística e Cognição. Juíz de Fora: Editora UFJF, pp. 109-119.

MURPHY, G. L. (1996 ). “On metaphoric representation.” In: Cognition 60, S. pp. 173-204.

NERLICH, B. \& CLARKE, D. D. (2001). "Mind, meaning and metaphor: the philosophy and psychology of metaphor in 19th-century Germany". In: History of the Human Sciences, 14(2), pp. 39-62.

NUESSEL, F. (1990). "More Than Cool Reason: A Field Guide to Poetic Metaphor". In: Metaphor and Symbol 5 (4), pp. 251-254.

PONTEROTTO, D. (2003). "The cohesive role of metaphor in discourse and conversation”. In: BARCELONA, A. Metaphor and metonymy at the crossroads: A cognitive perspective. Berlin, New York: Mouton de Gruyter, pp. 283-298.

QUINN, N. (1991). "The cultural basis of metaphor". In: FERNANDEZ, J. W. Beyond metaphor: The theory of tropes in anthropology. Stanford: Stanford University Press, pp. 56-93.

RICHARDS, I. A. (1936/1965). The Philosophy of Rhetoric. New York: Oxford University Press.

ROLF, E. (2005). Metapherntheorien. Typologie, Darstellung, Bibliographie. Berlin, New York: Walter de Gruyter.

ROSCH, E. (1978). "Principles of Categorization". In: ROSCH, E. \& LLOYD, B. B. Cognition and Categorization. Hillsdale: Lawrence Erlbaum Associates, pp. 27-48.

SARDINHA, T. B. (2007). Metáfora. São Paulo: Parábola Editorial.

SCHMITZ, H. W. (1985 ). „Die durchgängige Tropisierung der Sprache. Über einen Aspekt von, Zeichen im Wandel'“. In: DUTZ, K. D. \& SCHMITTER, P. Historiographia Semioticae. Studien zur Rekonstruktion der Theorie und Geschichte der Semiotik. Münster: MAkS Publikationen, pp. 241-270.

SCHRÖDER, U. (2009a). "Preferential Metaphorical Conceptualizations in Everyday Discourse About Love in the Brazilian and German Speech Communities". In: Metaphor and Symbol, 24/2, pp. 105-120.

. (2009b). "A mesclagem metafórica de Fauconnier \& Turner nas teorias de Karl Bühler e Wilhelm Stählin: antecipações e complementos". In: ABRALIN 40 Anos - VI Congresso Internacional da ABRALIN, 2009b, João Pessoa, Anais. João Pessoa: Idéia, 1, pp. 2913-2922.

. (2008a). "Antecipações da metáfora cotidiana nas concepções de Hans Blumenberg e Harald Weinrich". In: Revista de Estudos da Linguagem, 16, 2, pp. 39-54.

. (2008b). „Aufbrüche in der kognitiven Metaphernforschung aus kommunikationswissenschaftlicher Sicht“. In: ESCHBACH, A.; HALAWA, M. A. \& LOENHOFF, J. Audiator et altera pars. Kommunikationswissenschaft zwischen Historiographie, Theorie und empirischer Forschung. Aachen: Shaker Verlag, pp. 147-166.

. (2008c). "Da teoria cognitiva a uma teoria mais dinâmica, cultural e sociocognitiva da metáfora". In: Alfa (ILCSE/UNESP), v. 52, pp. 39-56.

. (2007). "Tendenzen gegenläufiger Reterritorialisierungen in brasilianischen und deutschen Rap-Texten". In: Lusorama, 71-72, pp. 93-119. 
. (2004). "Os precursores filosóficos da teoria cognitiva das metáforas". In: Cadernos de Estudos Lingüisticos 46/2, pp. 243-252.

SEMINO, E. (2008). Metaphor in Discourse. Cambridge: Cambridge University Press.

SILVA SINHA, V.; SINHA, C.; ZINKEN, J. \& SAMPAIO, W. (2009). "When Time is not Space: The social and linguistic construction of time intervals in an Amazonian culture." Journal of Pragmatics, in press.

SINHA, C. \& JENSEN DE LÓPEZ, K. (2000). "Language, culture and the embodiment of spatial cognition". In: Cognitive Linguistics, 11, pp. 17-41.

STÄHLIN, W. (1913). "Zur Psychologie und Statistik der Metaphern. Eine methodologische Untersuchung". Archiv für die gesamte Psychologie XXXI, Frankfurt, pp. 297-425.

STEEN, G. J. (2007). Finding Metaphor in Grammar and Usage. Amsterdam, Philadelphia: John Benjamins.

STEFANOWITSCH , A. \& GRIES, S. T. (2007). Corpus-Based Approaches to Metaphor and Metonymy. Berlin: Mouton de Gruyter.

VERVAEKE, J. \& KENNEDY, J. M. (1996). "Metaphors in language and thought: Falsification and multiple meanings". In: Metaphor and Symbol, 11, pp. 273-284.

ZANOTTO, M. S. \& PALMA, D. V. (2008). “Opening Pandora's box: Multiple readings of 'a metaphor". In: ZANOTTO, M. S.; CAMERON, L. \& CAVALCANTI, M. C. Confronting Metaphor in Use. An applied linguistic approach. Amsterdam, Philadelphia: John Benjamins, pp. 11-43.

ZINKEN, J. (2004). "Metphors, stereotypes, and the linguistic picture of the world: Impulses from the Ethnolinguistic School of Lublin." metaphorik.de, 7, pp. 115-136, http://www.metaphorik.de/07/zinken. pdf

ZINKEN, J. \& MUSOLFF, A. (2009). "Metaphorical Meadning \& Understanding". In: MUSOLFF, A. \& ZINKEN, J. Metaphor and Discourse. Hampshire: Palgrave Macmillan, pp. 1-8. 